\title{
REGULARITY RESULTS FOR DEGENERATE ELLIPTIC EQUATIONS RELATED TO GAUSS MEASURE
}

\author{
G. Di Blasio, F. FeO AND M. R. Posteraro
}

Abstract. In this paper we study a Dirichlet problem relative to the equation $L u=g \varphi-\left(f_{i} \varphi\right)_{x_{i}}$, where $L$ is a linear elliptic operator with lower-order terms whose ellipticity condition is given in terms of the function $\varphi(x)=(2 \pi)^{-\frac{n}{2}} \exp \left(-|x|^{2} / 2\right)$, the density of the Gaussian measure. We use the notion of rearrangement with respect to the Gauss measure to obtain a priory estimate of the solution $u$ and we study the summability of $u$ in the Lorentz-Zygmund spaces when $g$ and $f_{i}$ varies in suitable Lorent-Zygmund spaces.

Mathematics subject classification (2000): 35J70, 35B65.

Key words and phrases: Gauss measure, Smoothness and regularity of solution of PDE's, Elliptic PDE's of degenerate type.

\section{REFERENCES}

[1] R. A. ADAMs, General logarithmic Sobolev inequalities and Orlicz imbeddings, J. Funct. Anal., 34, (2) (1979), 292-303.

[2] A. Alvino, P. L. Lions AND G. TROMBetTI, Comparison results for elliptic and parabolic equations via Schwarz symmetrization, Ann. Inst. H. Poincarè Anal. non linéaire, 7, (1990), 37-65.

[3] A. Alvino, G. TROMBetTi, Sulle migliori costanti di maggiorazione per una classe di equazioni ellittiche degeneri, Ricerche Mat., 27, (1978), 413-428.

[4] A. Alvino, G. Trombetti, Equazioni ellittiche con termini di ordine inferiore e riordinamenti, Atti Accad. Naz. Lincei Cl. Sci. Fis. Mat. Nater. Rend. Lincei, 66, (8) (1979), 194-200.

[5] A. Alvino, G. Trombetti, Su una classe di equazioni ellittiche non lineari degeneri, Ricerche Mat., 29, (1980), 193-212.

[6] C. BANDLE, Isoperimetric Inequalities and applications, Pitman London, 1980.

[7] C. BennetT, K. Rudnick, On Lorentz-Zygmund spaces, Dissertationes Math. (Rozprawy Mat.), 175, (1980), 67.

[8] C. BenNetT, R. Sharpley, Interpolations of operator, Pure and Applied Mathematics, 129. Academic Press, Inc., Boston, MA, 1988.

[9] M. F. Betta, F. Brock, A. Mercaldo and M. R. Posteraro, A comparison result related to Gauss measure, C. R. Acad. Sci. Paris, 334, Serie I, (2002), 451-456.

[10] F. BETTA, Estimates for solutions of nonlinear degenerate elliptic equations, Att. Sem. Mat. Fis. Univ. Modena, XLV, (1997), 449-470.

[11] V. I. Bogachev, Gaussain measures, Mathematical Surveys and Monographs, 62. American Mathematical Society, Providence, RI, 1998.

[12] C. Borell, The Brunn-Minkowski inequality in the Gauss space, Invent. Math., 30, (1979), 194-200.

[13] F. CHIACCHIO, Comparison results for linear parabolic equations in unbounded domains via Gaussian symmetrization, DIE, 17, (3-4) (2004), 241-258.

[14] K. M. CHONG, N. M. RicE, Equimisurable rearrangements of Sobolev functions, J. Reine Angew. Math., 384, (1988), 153-179.

[15] G. DI BLASIO, Linear elliptic equations and Gauss measure, JIPAM. J. Inequal. Pure Appl. Math., 4, (5) (2003), Article 106, 11 pp. (electronic). 
[16] A. EHRHARD, Symmétrisation dans l'space de Gauss, Math. Scand. , 53, (1983), 281-301.

[17] A. EHRHARD, Inégalités isoperimétriques et intégrales de Dirichlet Gaussiennes, Ann. Scient. Éc. Norm. Sup., 4 e sèrie, t.17, (1984), 317-332.

[18] H. FEDERER, Geometric measure theory, Grundlehren der Mathematischen Wissenschaften 153, Springer-Verlag, 1969.

[19] A. FERONE, R. VOLPICELLI, Some relations between pseudo-rearrangement and relative rearrangement, Nonlinear Analysis, 41, (2000), 855-869.

[20] L. Gross, Logarithmic Sobolev inequalities, Amer. J. Math., 97, (1976), 1061-1083.

[21] M. LEDOUX, Isoperimetry and Gauss analysis, Lecture on probability theory and statictics (Saint-Flour, 1994), Lecture Notes in Math 1648, 164-294, Springer berlin, (1996).

[22] C. Miranda, Disuguaglianze, Lessons of Superior Mathematics held at the University of Naples in $1962 / 63$

[23] B. OPIC, L. PICK, On generalized Lorentz-Zygmund spaces, Math. Inequal. Appl., 2, (3) (1999), 391-467., 4 e sèrie, t. 17 (1984), 317-332

[24] M. K. W. MurTy, G. STAMPACCHIA, Boundary value problems for some degenerate elliptic operators, Ann. Mat. Pura Appl., 80, (1968), 1-122.

[25] J. M. RAKOTOSON, Some properties of the relative rearrangement, JMAA, 2, (135) (1988), 488-500.

[26] J. M. RAKOTOSON, B. SIMON, Relative rearrangement on a measure space. Application to the regularuty of weighted monotone rearrangement. Part1, Part2, Rev. R. Acad. Ciend. Exact. Fis. Nat. (Esp), 91, (1997), 17-31.

[27] G. TALENTI, Elliptic Equations and Rearrangements, Ann. Scuola Norm. Sup. Pisa Cl. Sci., 3, (1976), $697-718$.

[28] G. TALENTI, Nonlinear elliptic equation, rearrangements of functions and Orlicz spaces, Ann. Mat. Pura Appl., 120, (1979), 156-184.

[29] G. TALENTI, Linear Elliptic P.D.E.'s:Level Set, Rearrangements and a priori Estimates of Solutions, Boll. Un. Mat. Ital. B, 6 (4-B) (1985), 917-949.

[30] G. TALENTI, A weighted version of a rearrangement inequality, Ann. Univ. Ferrara, Sez. VII (NS), 43, (1997), 121-133.

[31] N. S. TRUDINGER, Linear elliptic operators with measurable coefficients, Ann. Scuola Norm. Sup. Pisa, 27, (1973), 265-308. 